\title{
An Audit of Cardiac Mortality Due to Acute Myocardial Infarction at a Tertiary Institution in the Southwestern Region of Trinidad and Tobago
}

\author{
$\mathrm{M}$ Bahall $^{1}$, T Seemungal ${ }^{2}$
}

\begin{abstract}
Objective: This study aims to identify the profile of patients who died from acute myocardial infarction (AMI) during hospital admission in southwest Trinidad.

Methods: This retrospective descriptive study was done using the death register of the San Fernando General Hospital (SFGH) during the period 2011 and 2012. Confirmed cases of AMI were selected based on the American College of Cardiology (ACC)/American Heart Association (AHA) guidelines 2011 or 2013. Relevant information on patient's profile, risks for ischaemic heart disease (IHD), clinical presentation and treatment were analysed using SPSS version 19 software.

Results: Males accounted for 56.3\% and females $43.8 \%$ of AMI deaths. East Indians were predominantly affected with an East Indian:African:Mixed ratio of 5:1:2. The mean age was 68.6 years and 72.8 years for males and females, respectively. The combination of diabetes mellitus and hypertension was responsible for most (52.5\%) AMI deaths. Patients who were not diabetic, hypertensive, smoking nor hypercholesterolaemic accounted for six (7.5\%) of the total deaths (4 = East Indians, $2=$ Mixed). One death occurred in the emergency department while the rest occurred in the ward; $44 \%$ of deaths occurred within 48 hours. Non-ST segment elevation myocardial infarction (NSTEMI) accounted for 70.0\% and STEMI for 30.0\% of AMI deaths. Five (20.8\%) of the 24 STEMI patients received thrombolytic treatment on arrival to the casualty department.

Conclusion: Deaths occurred predominantly among males of East Indian descent at a mean age of 70 years. The East Indian:African mortality ratio was 5:1. Patients suffered mainly from diabetes mellitus, hypertension or a combination of both.
\end{abstract}

Keywords: Diabetes mellitus, East Indian, hypertension, mortality, myocardial infarction, risk factors

WIMJ Open 2015; 2 (2): 60

\section{INTRODUCTION}

Cardiovascular (CVS) disease is a global phenomenon accounting for the leading cause of death worldwide (1). Coronary artery disease (CAD) affects about 13 million people in the United States of America (USA) and accounted for $36 \%$ of all deaths in 2002 (2). Worldwide, approximately $40 \%$ of all cardiovascular deaths are attributed to CAD, and it kills more than seven million people each year (3). Cardiovascular disease is a major cause of morbidity and its prevalence is increasing because of the increasing burden of cardiovascular risk factors such as diabetes mellitus (DM), hypertension (HTN), hypercholesterolaemia, smoking, alco-

From: ${ }^{1}$ Department of Medicine, San Fernando General Hospital, San Fernando, Trinidad and Tobago and ${ }^{2}$ Department of Clinical Medical Sciences, Faculty of Medical Sciences, The University of the West Indies, St Augustine, Trinidad and Tobago.

Correspondence: Dr M Bahall, Lp 62, Calcutta Rd No 3, McBean, Couva, Trinidad and Tobago, West Indies. E-mail: vmandrakes@hotmail.com hol, obesity and sedentary lifestyle. Both the INTERHEART study (4) and the Framingham study $(5,6)$, expanded the risk factors to include waist/hip ratio, dietary patterns, physical activity, blood apolipoproteins (Apo) and psychosocial factors. In addition, the large East Indian migrant population is thought to pose an additional risk factor (7). Trinidad and Tobago, with its cosmopolitan composition, provides a unique social setting to study and analyse risk factors.

Trinidad and Tobago comprises a population that is predominantly East Indian (35.4\%), African (34.2\%) and mixed [22.8\%] (8). However, in South Trinidad, the population comprises 294461 East Indians and 133918 Africans [East Indian:African ratio is 2.2:1] (8). The incidence of deaths from cardiovascular disease in Trinidad and Tobago is about 34\% (9). Barcelo claims that deaths attributed to cardiovascular disease in the Caribbean and Latin America will increase by more than $60 \%$ between 2000 and 2020 if preventative measures are not implemented (10). Studies on sudden death have revealed CAD is a significant cause of 
sudden cardiac death. In Jamaica, with a predominantly African population, $7 \%$ of sudden natural deaths resulted from ischaemic heart disease but this was only in patients who had postmortems (11), while in rural south India, cardiovascular disease accounted for $75 \%$ of sudden cardiac deaths (12). A study of coroner's autopsies in Benin City, Nigeria, revealed that the most common cause of sudden unexpected natural death were cardiovascular diseases resulting from complications of HTN [54.7\%] (13). Acute myocardial infarction mortality in Cuba was 18.3\% (14). An audit of sudden deaths at one Accident and Emergency (A\&E) Department in north Trinidad found that AMI was responsible for $27.35 \%$ of deaths, with AfroTrinidadians accounting for $59 \%$ of deaths due to AMI (15). However, there is no information on deaths from AMI in south Trinidad. The aetiology of this alarming AMI mortality rate at presentation is of major concern to individuals and policy-makers.

We reviewed the deaths of patients from AMI during their admission at the San Fernando General Hospital (SFGH), which is the tertiary care institution for nearly all AMI patients in south Trinidad, with the aim of determining patient profile, risk factors and complications of patients who die from AMI after admission to the medical department.

\section{SUBJECTS AND METHODS}

The total annual admissions at SFGH for 2011-2012 was 52 864 and total medical admissions was 18052 (ie 34\% of total admissions). This has been the general pattern for years. The ethnic composition of all admissions to SFGH during the study period was not ascertained; however, the ethnic composition for all admissions for the month of April 2015 was East Indians, 59.7\%, Africans, 29.2\%, mixed, 8.3\%, Chinese, $0.3 \%$ and other, $2.5 \%$. Medical department admissions were not readily available. However, using names as a guide, patients admitted to the medical department with East Indian names was 55\%. The ethnic composition of any month can reflect an approximation for the 2011-2012 study period.

The registry of deaths collated by the registry of births and deaths department at the SFGH was used to obtain cases of AMI deaths during the period 2011 and 2012. Data were collected on patient profile, presenting symptoms, compli- cations, risk factors, type of AMI and treatment received. Files of all $(n=183)$ patients whose cause of death was registered as AMI were reviewed. Inclusion criteria included admitted cases of AMI that fulfilled the diagnostic criteria. Exclusion criteria included all doubtful cases. Deaths on arrival were excluded since these cases were not under the care of the admitting medical unit. These cases (some would have died from AMI) were transferred to the mortuary for post mortem examination. Sixty-three cases were confirmed by a detection of a rise and/or fall of cardiac biomarkers (troponin $\geq 0.1$ ) with evidence of ischaemia with at least one of the following: symptoms of ischaemia, electrocardiogram (ECG) changes [new ST-T changes, left bundle branch block or pathological Q waves] $(16,17)$. Seventeen cases had no troponin levels for review. However, nine of these cases had significant ST depression with raised creatine kinase (CK) levels (non-ST segment elevation myocardial infarction; NSTEMI): five had moderate CK elevation (1000 to over 4000) and four had mild CK elevation. Eight cases had significant ST elevation with raised CK levels and were accepted as STEMI. Post mortem examination was not conducted since there was sufficient evidence for the diagnosis of AMI. All information taken from the patient's file was kept confidential and stored in a computer database using a unique patient identification code. Data which were accessible only to the researchers were analysed using SPSS version 19 software. Simple descriptive analysis was done.

\section{RESULTS}

Out of 183 cases registered as AMI, 80 cases were confirmed as AMI. The other 103 cases were excluded since they did not fulfil the criteria for AMI. Table 1 shows the distribution of AMI deaths in the different age groups. The mean age at death was 70.5 (range: 40 to 93 ) years. The mean (range) age for males was 68.6 (40-92) years, whereas the mean (range) age for females was 72.8 (49-93) years. There were 45 $(56.3 \%)$ males and $35(43.8 \%)$ females; the majority, 51 $(63.7 \%)$ were East Indians, 10 (12.5\%) were Africans and 19 (23.8\%) were mixed. Acute myocardial infarction mortality among East Indians was five times that of Africans. The 103 patients excluded comprised $58(56.3 \%)$ East Indians, 21 (20.4\%) Africans and 24 (23.3\%) mixed. Non-ST segment elevation myocardial infarction accounted for $70.0 \%$ and

Table 1: Acute myocardial infarction patients by age and ethnicity

\begin{tabular}{|c|c|c|c|c|c|c|c|}
\hline \multicolumn{2}{|l|}{ Variables } & Male (\%) & $95 \%$ CI & Female (\%) & $95 \%$ CI & Total (\%) & $95 \%$ CI \\
\hline \multicolumn{2}{|c|}{ Sample size } & $45(56.3)$ & $45.0,67.5$ & $35(43.8)$ & $32.5,55.0$ & $80(100.0)$ & \\
\hline \multicolumn{8}{|l|}{ Age group } \\
\hline \multirow[t]{4}{*}{ (years): } & $36-49$ & $4(5.0)$ & $2.3,18.2$ & $1(1.3)$ & $0.0,8.6$ & $5(6.3)$ & $1.3,12.6$ \\
\hline & $50-65$ & $12(15.0)$ & $15.9,40.9$ & $6(7.5)$ & $5.7,31.4$ & $18(22.5)$ & $13.9,32.9$ \\
\hline & $66-75$ & $12(15.0)$ & $13.6,40.9$ & $16(20.0)$ & $31.4,62.9$ & $28(35.0)$ & $25.3,45.6$ \\
\hline & $>75$ & $16(20.0)$ & $22.7,50.0$ & $12(15.0)$ & $20.0,51.4$ & $28(35.0)$ & $25.3,45.6$ \\
\hline \multirow[t]{3}{*}{ Ethnicity: } & African & $3(3.8)$ & $55.6,82.2$ & $7(8.8)$ & $40.0,74.3$ & $10(12.5)$ & $52.5,75.0$ \\
\hline & East Indian & $31(38.8)$ & $0.0,15.6$ & $20(25.0)$ & $8.6,34.3$ & $51(63.7)$ & $6.3,20.0$ \\
\hline & Mixed & $11(13.8)$ & $13.3,37.8$ & $8(10.0)$ & $8.6,37.1$ & 19 (23.8) & $15.0,33.7$ \\
\hline
\end{tabular}


STEMI $30.0 \%$ of deaths. Nearly all patients, 78 or $97.5 \%$, utilized the public Emergency Medical Service (EMS); two $(2.5 \%)$ used private transportation. Table 2 gives the profile of patients between males and females.

Most ( $\mathrm{n}=49 ; 61.3 \%)$ had a pulse less than or equal to 100 beats per minute at presentation. The mean (range) pulse was $92.2(42-152)$ beats per minute. Systolic blood pressure of patients was $140 \mathrm{mmHg}(62.5 \%, \mathrm{n}=50), 140-160(17.5 \%$, $\mathrm{n}=14)$, and over $160(20.0 \%, \mathrm{n}=16)$, while the mean (range) systolic blood pressure reading was 134.9 (60-268). On arrival at the emergency department, $41.3 \%$ had acute pulmonary oedema. Other complications included cardiac arrest and cardiogenic shock. Seventy-nine (98.8\%) patients died on the ward and one patient (1.3\%) died in the emergency department. Nine (11.3\%) patients died within 24 hours of arrival while $25(31.3 \%)$ died within one to two

Table 2: Acute myocardial infarction patient characteristics by gender

\begin{tabular}{|c|c|c|c|c|c|c|}
\hline Variables & Male $(n=45)$ & SE (\%) & $95 \% \mathrm{CI}$ & Female $(\mathrm{n}=35)$ & SE (\%) & $95 \%$ CI \\
\hline Mean age (years) & 68.6 & & & 72.8 & & \\
\hline Most common age group & $>75$ & 7.2 & $22.7,50.0$ & $65-75$ & 8.4 & $31.4,62.9$ \\
\hline \multicolumn{7}{|l|}{ Ethnicity } \\
\hline African & $6.7 \%(\mathrm{n}=3)$ & 3.7 & $0.0,15.6$ & $20.0 \%(\mathrm{n}=7)$ & 6.6 & $8.6,34.3$ \\
\hline East Indian & $68.9 \%(\mathrm{n}=31)$ & 7.0 & $55.6,82.2$ & $57.1 \%(\mathrm{n}=20)$ & 8.8 & $40.0,74.3$ \\
\hline Mixed & $24.4 \%(\mathrm{n}=11)$ & 6.4 & $13.3,37.8$ & $22.9 \%(\mathrm{n}=8)$ & 7.3 & $8.6,37.1$ \\
\hline Use of EMS for transportation & $97.8 \%(n=44)$ & 2.3 & $93.3,100.0$ & $97.1 \%(n=34)$ & 2.9 & $91.4,100.0$ \\
\hline $\begin{array}{l}\text { Modal day range alive after } \\
\text { chest pain }\end{array}$ & $1-2$ & 6.6 & $17.8,42.2$ & $1-2$ & 7.6 & $17.1,45.7$ \\
\hline \multicolumn{7}{|l|}{ Clinical presentation } \\
\hline Death on ward & $100.0 \%(\mathrm{n}=45)$ & 0.0 & $100.0,100.0$ & $97.1 \%(n=34)$ & 2.8 & $0.0,8.6$ \\
\hline Death in emergency area & $0.0 \%(\mathrm{n}=0)$ & 0.0 & $0.0,0.0$ & $2.9 \%(\mathrm{n}=1)$ & 2.8 & $91.4,100.0$ \\
\hline Death caused by STEMI & $26.7 \%(\mathrm{n}=12)$ & 6.6 & $13.3,40.0$ & $34.3 \%(\mathrm{n}=12)$ & 8.2 & $20.0,51.4$ \\
\hline Death caused by NSTEMI & $73.3 \%(n=33)$ & 6.6 & $60.0,86.7$ & $65.7 \%(\mathrm{n}=23)$ & 8.2 & $48.6,80.0$ \\
\hline Systolic blood pressure & & & & & & \\
\hline$<140$ & $66.7 \%(\mathrm{n}=30)$ & 7.3 & $53.3,80.0$ & $57.1 \%(\mathrm{n}=20)$ & 8.2 & $40.0,74.3$ \\
\hline$>160$ & $15.6 \%(\mathrm{n}=7)$ & 5.2 & $4.4,24.4$ & $25.7 \%(\mathrm{n}=9)$ & 5.9 & $2.9,25.7$ \\
\hline Pulse: $>100$ beats per minute & $37.8 \%(\mathrm{n}=17)$ & 7.2 & $24.4,51.1$ & $40.0 \%(\mathrm{n}=14)$ & 8.5 & $22.9,57.1$ \\
\hline Lungs: Creps & $35.6 \%(n=16)$ & 7.4 & $22.2,48.9$ & $48.6 \%(n=17)$ & 7.4 & $31.5,65.7$ \\
\hline \multicolumn{7}{|l|}{ State of consciousness } \\
\hline Conscious & $24.4 \%(\mathrm{n}=11)$ & 6.5 & $13.3,37.8$ & $22.9 \%(n=8)$ & 7.1 & $8.6,37.1$ \\
\hline Semi-conscious & $75.6 \%(\mathrm{n}=34)$ & 6.5 & $62.2,86.7$ & $74.3 \%(n=26)$ & 7.3 & $60.0,88.6$ \\
\hline Unconscious & $0.0 \%(\mathrm{n}=0)$ & 0.0 & $0.0,0.0$ & $2.9 \%(\mathrm{n}=1)$ & 2.9 & $0.0,8.6$ \\
\hline Blood test conducted & $97.8 \%(\mathrm{n}=44)$ & 2.2 & $93.3,100.0$ & $100.0 \%(\mathrm{n}=35)$ & 0.0 & $100.0,100.0$ \\
\hline \multicolumn{7}{|l|}{ Blood test results } \\
\hline Moderate CK (200-1000) & $42.2 \%(n=19)$ & 8.2 & $34.2,65.8$ & $37.1 \%(n=13)$ & 9.1 & $22.6,58.1$ \\
\hline High CK (> 1000) & $26.7 \%(\mathrm{n}=12)$ & 7.5 & $18.4,47.4$ & $28.6 \%(n=10)$ & 8.2 & $16.1,48.4$ \\
\hline High cholesterol $(\geq 200)$ & $20.0 \%(n=9)$ & 7.8 & $12.9,45.2$ & $25.7 \%(\mathrm{n}=9)$ & 9.3 & $21.7,56.5$ \\
\hline \multicolumn{7}{|l|}{ Treatment } \\
\hline Thrombolysis & $33.3 \%(\mathrm{n}=15)$ & 7.1 & $17.8,46.7$ & $11.4 \%(n=4)$ & 5.5 & $2.9,22.9$ \\
\hline Oxygen & $97.8 \%(\mathrm{n}=44)$ & 2.1 & $93.3,100.0$ & $94.3 \%(\mathrm{n}=33)$ & 3.9 & $85.7,100.0$ \\
\hline Aspirin & $80.0 \%(\mathrm{n}=36)$ & 5.9 & $66.7,91.1$ & $68.6 \%(\mathrm{n}=24)$ & 7.7 & $54.3,82.9$ \\
\hline Plavix (clopidogrel) & $55.6 \%(\mathrm{n}=25)$ & 7.6 & $40.0,71.1$ & $62.9 \%(n=22)$ & 8.2 & $45.7,80.0$ \\
\hline Clexane & $53.3 \%(\mathrm{n}=24)$ & 7.6 & $37.8,68.9$ & $68.6 \%(n=24)$ & 7.9 & $51.5,82.9$ \\
\hline Beta blocker & $33.3 \%(\mathrm{n}=15)$ & 6.9 & $22.2,46.7$ & $28.6 \%(n=10)$ & 7.4 & $14.3,42.9$ \\
\hline Cholesterol drug & $46.7 \%(\mathrm{n}=21)$ & 7.4 & $33.3,62.2$ & $51.4 \%(\mathrm{n}=18)$ & 8.5 & $57.1,85.7$ \\
\hline \multicolumn{7}{|l|}{ Risk factors } \\
\hline Diabetes mellitus (DM) & $57.8 \%(\mathrm{n}=26)$ & 7.5 & $42.2,73.3$ & $74.3 \%(\mathrm{n}=26)$ & 7.7 & $60.0,88.6$ \\
\hline Hypertension (HTN) & $62.2 \%(\mathrm{n}=28)$ & 7.3 & $46.7,77.8$ & $77.1 \%(\mathrm{n}=27)$ & 6.9 & $62.9,88.6$ \\
\hline $\mathrm{DM}+\mathrm{HTN}$ & $44.4 \%(n=20)$ & 7.3 & $31.1,57.8$ & $62.9 \%(n=22)$ & 8.4 & $45.7,80.0$ \\
\hline Hypercholesterolaemia & $2.2 \%(\mathrm{n}=1)$ & 2.1 & $0.0,6.7$ & $8.6 \%(n=3)$ & 4.8 & $0.0,20.0$ \\
\hline End-stage renal disease & $2.2 \%(\mathrm{n}=1)$ & 2.2 & $0.0,6.7$ & $2.9 \%(\mathrm{n}=1)$ & 2.8 & $0.0,8.6$ \\
\hline Smoking & $24.4 \%(\mathrm{n}=11)$ & 6.2 & $13.3,37.8$ & $11.4 \%(n=4)$ & 5.3 & $2.9,22.9$ \\
\hline Ischaemic heart disease & $11.1 \%(n=5)$ & 4.7 & $2.2,22.2$ & $22.9 \%(n=8)$ & 7.1 & $8.6,37.1$ \\
\hline Other risk factors & $28.9 \%(\mathrm{n}=13)$ & 6.7 & $15.6,42.2$ & $8.6 \%(\mathrm{n}=3)$ & 4.7 & $0.0,20.0$ \\
\hline
\end{tabular}

EMS: emergency medical service; NSTEMI: non-ST-segment elevation myocardial infarction; CK: creatine kinase 
days, $15(18.8 \%)$ within two to three days, five $(6.3 \%)$ within three to four days, $11(13.8 \%)$ within four to five days and 15 (18.8\%) after more than five days.

Risk factors identified included HTN $(68.8 \%, \mathrm{n}=55)$, DM $(65.0 \%, \mathrm{n}=52)$, hypercholesterolaemia $(5.0 \%, \mathrm{n}=4)$, end-stage renal disease (ESRD; $2.5 \%, \mathrm{n}=2$ ), chronic renal failure (CRF) except ESRD $(20.0 \%, \mathrm{n}=16)$, and smoking $(18.8 \%, \mathrm{n}=15)$. Information on obesity, alcohol and stress, diet and exercise were not recorded. Table 3 shows that the oedema and all were semi-conscious. The data also showed that based on results from 54 patients, 20 (37.0\%) had a total cholesterol of greater than $200 \mathrm{mg} / \mathrm{dL}$.

Among the East Indian sample of AMI deaths, 32 (62.7\%) had DM whilst 33 (64.7\%) were hypertensive; 26 $(51.0 \%)$ had a combination of both DM and HTN. This combination also accounted for six (60.0\%) AMI deaths among Africans and $10(52.6 \%)$ of the mixed population. Of the six $(7.5 \%)$ patients who were not diabetic, hypertensive,

Table 3: Risk factors among patients who died from acute myocardial infarction

\begin{tabular}{lccc}
\hline Risk factors & Male $(\mathbf{n}=\mathbf{4 5})$ & Female $(\mathbf{n}=\mathbf{3 5})$ & Total $(\mathbf{n}=\mathbf{8 0})$ \\
\hline Diabetes mellitus & $57.8 \%(\mathrm{n}=26)$ & $74.3 \%(\mathrm{n}=26)$ & $65.0 \%(\mathrm{n}=52)$ \\
Hypertension & $62.2 \%(\mathrm{n}=28)$ & $77.1 \%(\mathrm{n}=27)$ & $68.8 \%(\mathrm{n}=55)$ \\
Smoking & $24.4 \%(\mathrm{n}=11)$ & $11.4 \%(\mathrm{n}=4)$ & $18.8 \%(\mathrm{n}=15)$ \\
Hypercholesterolaemia & $22.2 \%(\mathrm{n}=10)$ & $28.6 \%(\mathrm{n}=10)$ & $25.0 \%(\mathrm{n}=20)$ \\
DM + HTN & $44.4 \%(\mathrm{n}=20)$ & $62.9 \%(\mathrm{n}=22)$ & $52.5 \%(\mathrm{n}=42)$ \\
DM + SM & $13.3 \%(\mathrm{n}=6)$ & $8.6 \%(\mathrm{n}=3)$ & $11.3 \%(\mathrm{n}=9)$ \\
DM + HC & $11.1 \%(\mathrm{n}=5)$ & $25.7 \%(\mathrm{n}=9)$ & $17.5 \%(\mathrm{n}=14)$ \\
HTN + SM & $13.3 \%(\mathrm{n}=6)$ & $11.4 \%(\mathrm{n}=4)$ & $12.5 \%(\mathrm{n}=10)$ \\
HTN + HC & $15.6 \%(\mathrm{n}=7)$ & $22.9 \%(\mathrm{n}=8)$ & $18.8 \%(\mathrm{n}=15)$ \\
SM + HC & $8.9 \%(\mathrm{n}=4)$ & $5.7 \%(\mathrm{n}=2)$ & $7.4 \%(\mathrm{n}=6)$ \\
DM + HTN + SM & $8.9 \%(\mathrm{n}=4)$ & $8.6 \%(\mathrm{n}=3)$ & $8.8 \%(\mathrm{n}=7)$ \\
DM + HTN + HC & $8.9 \%(\mathrm{n}=4)$ & $22.9 \%(\mathrm{n}=8)$ & $15.0 \%(\mathrm{n}=12)$ \\
DM + SM + HC & $6.7 \%(\mathrm{n}=3)$ & $5.7 \%(\mathrm{n}=2)$ & $6.3 \%(\mathrm{n}=5)$ \\
HTN + SM + HC & $6.7 \%(\mathrm{n}=3)$ & $5.7 \%(\mathrm{n}=2)$ & $6.3 \%(\mathrm{n}=5)$ \\
DM + HTN + SM + HC & $6.7 \%(\mathrm{n}=3)$ & $5.7 \%(\mathrm{n}=2)$ & $6.3 \%(\mathrm{n}=5)$ \\
Neither DM/HTN/SM/HC & $8.9 \%(\mathrm{n}=4)$ & $5.7 \%(\mathrm{n}=2)$ & $7.5 \%(\mathrm{n}=6)$ \\
\hline
\end{tabular}

$\mathrm{DM}=$ diabetes mellitus, $\mathrm{HTN}=$ hypertension, $\mathrm{SM}=$ smoking, $\mathrm{HC}=$ hypercholesterolaemia

majority of AMI deaths resulted from patients suffering from $\operatorname{HTN}(68.8 \%, \mathrm{n}=55)$, followed by DM $(65.0 \%, \mathrm{n}=52)$ and then a combination of DM and $\operatorname{HTN}(52.5 \%, n=42)$. Six (7.5\%) patients did not have DM, HTN, hypercholesterolemia, nor did they smoke. Of these six patients, it was revealed that one (16.7\%) had CRF, one (16.7\%) had a massive gastrointestinal bleed, three $(50.0 \%)$ had pulmonary hypercholesterolaemic nor smokers, four were East Indians and two were mixed (Table 4).

Five $(20.8 \%)$ of the 24 STEMI patients received thrombolytic therapy on arrival to casualty. Other treatment received either in the emergency department or on the wards included aspirin $-60(75.0 \%)$, glyceryl trinitrate (GTN) -42 $(52.5 \%)$, oxygen $-77(96.3 \%)$, morphine - 30 (37.5\%), betablocker - $25(31.3 \%)$, clexane - $48(60.0 \%)$, cholesterol drug

Table 4: Risk factors by ethnicity among patients who died from acute myocardial infarction

\begin{tabular}{lcccc}
\hline Risk factors & $\begin{array}{c}\text { African } \\
(\mathbf{n}=\mathbf{1 0})\end{array}$ & $\begin{array}{c}\text { East Indian } \\
(\mathbf{n}=\mathbf{5 1})\end{array}$ & $\begin{array}{c}\text { Mixed } \\
(\mathbf{n}=\mathbf{1 9})\end{array}$ & $\begin{array}{c}\text { Total } \\
(\mathbf{n}=\mathbf{8 0})\end{array}$ \\
\hline Diabetes mellitus & $60.0 \%(\mathrm{n}=6)$ & $62.7 \%(\mathrm{n}=32)$ & $73.7 \%(\mathrm{n}=14)$ & $65.0 \%(\mathrm{n}=52)$ \\
Hypertension & $90.0 \%(\mathrm{n}=9)$ & $64.7 \%(\mathrm{n}=33)$ & $68.4 \%(\mathrm{n}=13)$ & $68.8 \%(\mathrm{n}=55)$ \\
Smoking & $0.0 \%(\mathrm{n}=0)$ & $23.5 \%(\mathrm{n}=12)$ & $15.8 \%(\mathrm{n}=3)$ & $18.8 \%(\mathrm{n}=15)$ \\
Hypercholesterolemia & $20.0 \%(\mathrm{n}=2)$ & $31.4 \%(\mathrm{n}=16)$ & $10.5 \%(\mathrm{n}=2)$ & $25.0 \%(\mathrm{n}=20)$ \\
DM + HTN & $60.0 \%(\mathrm{n}=6)$ & $51.0 \%(\mathrm{n}=26)$ & $52.6 \%(\mathrm{n}=10)$ & $52.5 \%(\mathrm{n}=42)$ \\
DM + SM & $0.0 \%(\mathrm{n}=0)$ & $13.7 \%(\mathrm{n}=7)$ & $10.5 \%(\mathrm{n}=2)$ & $11.3 \%(\mathrm{n}=9)$ \\
DM + HC & $20.0 \%(\mathrm{n}=2)$ & $19.6 \%(\mathrm{n}=10)$ & $10.5 \%(\mathrm{n}=2)$ & $17.5 \%(\mathrm{n}=14)$ \\
HTN + SM & $0.0 \%(\mathrm{n}=0)$ & $13.7 \%(\mathrm{n}=7)$ & $15.8 \%(\mathrm{n}=3)$ & $12.5 \%(\mathrm{n}=10)$ \\
HTN + HC & $20.0 \%(\mathrm{n}=2)$ & $21.6 \%(\mathrm{n}=11)$ & $10.5 \%(\mathrm{n}=2)$ & $18.8 \%(\mathrm{n}=15)$ \\
SM + HC & $0.0 \%(\mathrm{n}=0)$ & $7.8 \%(\mathrm{n}=4)$ & $10.5 \%(\mathrm{n}=2)$ & $7.4 \%(\mathrm{n}=6)$ \\
DM + HTN + SM & $0.0 \%(\mathrm{n}=0)$ & $9.8 \%(\mathrm{n}=5)$ & $10.5 \%(\mathrm{n}=2)$ & $8.8 \%(\mathrm{n}=7)$ \\
DM + HTN + HC & $20.0 \%(\mathrm{n}=2)$ & $15.7 \%(\mathrm{n}=8)$ & $10.5 \%(\mathrm{n}=2)$ & $15.0 \%(\mathrm{n}=12)$ \\
DM + SM + HC & $0.0 \%(\mathrm{n}=0)$ & $5.9 \%(\mathrm{n}=3)$ & $10.5 \%(\mathrm{n}=2)$ & $6.3 \%(\mathrm{n}=5)$ \\
HTN + SM + HC & $0.0 \%(\mathrm{n}=0)$ & $5.9 \%(\mathrm{n}=3)$ & $10.5 \%(\mathrm{n}=2)$ & $6.3 \%(\mathrm{n}=5)$ \\
DM + HTN + SM + HC & $0.0 \%(\mathrm{n}=0)$ & $5.9 \%(\mathrm{n}=3)$ & $10.5 \%(\mathrm{n}=2)$ & $6.3 \%(\mathrm{n}=5)$ \\
Neither DM/HTN/SM/HC & $0.0 \%(\mathrm{n}=0)$ & $7.8 \%(\mathrm{n}=4)$ & $10.5 \%(\mathrm{n}=2)$ & $7.5 \%(\mathrm{n}=6)$ \\
\hline
\end{tabular}

$\mathrm{DM}=$ diabetes mellitus, $\mathrm{HTN}=$ hypertension, $\mathrm{SM}=$ smoking, $\mathrm{HC}=$ hypercholesterolaemia 
$-39(48.8 \%)$, insulin $-28(35.0 \%)$, clopidogrel -47 (58.8\%), angiotensin-converting enzyme (ACE) inhibitor - 34 $(42.5 \%)$, trimetazidine $-25(31.3 \%)$ and isosorbide mononitrate - $33(41.3 \%)$.

Investigations done were ECG - 78 (97.5\%) patients, chest X-ray - 58 (72.5\%), computed tomography (CT) scan - $15(18.8 \%)$ and an echocardiogram - nine (11.3\%) patients. As seen in Table 5, three inflammatory markers (troponin T, white blood cells and C-reactive protein) were documented. Troponin $\mathrm{T}$ levels were documented from 63 patients: one had a peak troponin level of 0.093 which was approximated to 0.1 , fourteen had troponin $\mathrm{T}$ levels equal to 0.1 , twenty-six (41.3\%) between 0.11 and 2.0 , sixteen $(25.4 \%)$ between 2.1 and 10.0 and six $(9.5 \%)$ had levels greater than 10.0.

Table 5: Peak cardiac biomarkers values of acute myocardial infarction (AMI) patients

\begin{tabular}{ccc}
\hline Test Range & $\begin{array}{c}\text { Number of MI } \\
\text { patients }\end{array}$ & $\begin{array}{c}\text { Per cent of MI } \\
\text { patients }\end{array}$ \\
\hline Troponin T & $\mathbf{6 3}$ & $\mathbf{1 0 0 . 0 \%}$ \\
$\leq 0.1$ & 15 & 23.8 \\
$0.11-2$ & 26 & 41.3 \\
$2.1-10$ & 16 & 25.4 \\
$>10$ & 6 & 9.5 \\
White blood cells & $\mathbf{7 2}$ & $\mathbf{1 0 0 . 0} \%$ \\
$<11$ & 29 & 40.3 \\
$11.1-20$ & 34 & 47.2 \\
$20.1-40$ & 9 & 12.5 \\
C-reactive protein & $\mathbf{3 8}$ & $\mathbf{1 0 0 . 0} \%$ \\
$<1.0$ & 6 & 15.8 \\
$1.0-3.0$ & 2 & 5.3 \\
$>3.0$ & 30 & 78.9 \\
\hline
\end{tabular}

\section{DISCUSSION}

This study was conducted in a tertiary healthcare hospital in south Trinidad. We found that deaths among East Indians were disproportionately higher than in Africans, 5.1:1, even though there is a 2.2:1 ratio between East Indians and Africans in south Trinidad (8). Amongst patients dying with AMI, DM and HTN were present in roughly equal proportions among the East Indians, $62.7 \%$ and $64.7 \%$, respectively. Hypertension was more common (90\%) among the Africans, and the combination of DM and HTN was associated with $51 \%$ of deaths among East Indians and $60 \%$ among Africans. Deaths from AMI showed significant differences by age, gender and ethnicity. Deaths were more common in the elderly, with females older than males. The mean age (68.6 years) for males is four years less than females (72.8 years). The majority $(70 \%)$ of deaths were NSTEMIs. Ambulance was the favoured means of transport with $98 \%$ of patients using it to get to hospital.

Deaths from AMI continue to be a major problem. There are limited studies on AMI mortality in Trinidad and the rest of the West Indies. In England, deaths per 100000 decreased in men from 78.7 (95\% confidence interval (CI) $77.7,79.8)$ in 2002 to $39.2(95 \%$ CI $38.6,39.9)$ in 2010 , and in women decreased per 100000 from 37.3 (95\% CI 36.8, $37.9)$ to 17.7 [95\% CI 17.4, 18.1] (18). Cardiovascular disease is the leading cause of death in Trinidad and Tobago (19) and its incidence is rising (8). Beharry et al (15) reported that $27.7 \%$ of $A \& E$ deaths resulted from AMI at a tertiary institution in Trinidad and Tobago. Deaths from AMI in Trinidad continue to increase because of the increasing burden of modifiable risk factors which were identified in the St James study (20) and the study of risk factors by Thomas et al (21).

Though there are concerns about the use of public transport including the public ambulance, nearly all patients utilized such service. The cause of this was not scrutinized, although this may result from patient's unwillingness to use private transport, cost or even poor evaluation of the health situation. There may also be unreasonable delays in calling for help or delayed response by the ambulance service. Twenty per cent of eligible STEMI patients were thrombolysed. Furthermore, there was a significant number of patients who had not received aspirin, clopidogrel and enoxaparin (standard treatments for acute coronary syndrome).

This study was conducted at a tertiary hospital in south Trinidad which services a population of predominantly East Indians. Though the sample was small, there is important information concerning AMI deaths. Other limitations of the study include the absence of clear documentation of all risk factors, and results of inflammatory markers. Many of the AMI deaths might have been omitted because of inadequate information such as cardiac biomarkers and ECG. Deaths prior to admission or on arrival were excluded since only admitted patients were included in the sample.

The East Indian population is particularly at risk. However, because DM and HTN are very common among both East Indians and Africans in this MI mortality population, every effort must be made to minimize these risks through change in modifiable risks such as healthier lifestyle and better control of DM and HTN. There may be other risk factors such as diet and exercise but these were not documented in the admission notes.

The study shows that AMI mortality of admitted patients is much more common among the East Indian population. The average age of death (70.5 years) is not much different from the average life expectancy. It is quite instructive that the vast majority (98\%) of patients opted to use the public ambulance transport and thrombolysis was undertaken in only $20 \%$ of eligible patients. Both DM and HTN are major determinants to AMI deaths.

Studies of this type will continue to unravel the health dominants of AMI and also understand the gaps in healthcare. This will assist in fashioning public health policy.

\section{REFERENCES}

1. Gersh BJ, Sliwa K, Mayosi BM, Yusuf S. The epidemic of cardiovascular disease in the developing world: global implications [Internet]. Eur Heart J 2010 [cited 2014 Feb 3]; 6: 642-8. Available from http:// eurheartj.oxfordjournals.org/content/31/6/642.full.pdf 
2. Knox J, Gaster B. Dietary supplements for the prevention and treatment of coronary artery disease. J Altern Complement Med 2007; 13: 83-95.

3. Wood MJ, Stewart RL, Merry H, Johnstone DE, Cox JL. Use of complementary and alternative medical therapies in patients with cardiovascular disease. Am Heart J 2003; 145: 806-12.

4. Yusuf S, Hawken S, Ounpuu S, Dans T, Avezum A, Lanas F et al; on behalf of the INTERHEART Study Investigators. Effect of potentially modifiable risk factors associated with myocardial infarction in 52 countries (the INTERHEART study): case-control study. Lancet 2004; 364: $937-52$.

5. O'Donnell C, Elosua R. Cardiovascular risk factors. Insights from Framingham Heart Study [Internet]. Rev Esp Cardiol 2008 [cited 2014 Feb 3]; 61: 299-310. DOI: 10.1157/13116658. Available from: http:// www.revespcardiol.org/es/factores-riesgo-cardiovascular-perspectivasderivadas/articulo/13116658/

6. Ryoo J-H, Cho SH, Kim S-W. Prediction of risk factors for coronary heart disease using Framingham Risk Score in Korean men [Internet]. PLoS ONE 2012 [cited 2014 Feb 3]; 7: e45030. doi: 10.1371/ journal.pone.0045030. Available from: http://www.plosone.org/article/ fetchObject.action?uri=info\%3Adoi\%2F10.1371\%2Fjournal.pone.004 5030\&representation $=\mathrm{PDF}$

7. Gupt M, Brister S. Is South Asian ethnicity an independent cardiovascular risk factor? Can J Cardiol 2006; 22: 194-7.

8. Trinidad and Tobago Central Statistical Office. Trinidad and Tobago 2011 population and housing census demographic report [Internet]; Port-of-Spain: Central Statistical Office; 2011 [cited 2013 Dec 12]. Available from: http://cso.planning.gov.tt/sites/default/files/content/ images/census/TRINIDAD\%20AND\%20TOBAGO\%202011\%20Dem ographic\%20Report.pdf

9. World Health Organization. Non-communicable diseases country profiles 2011 [Internet]. Geneva: WHO; 2011 [cited 2014 Feb 3]: 189. Available from: http://whqlibdoc.who.int/publications/2011/97892 41502283 eng.pdf?ua=1www.ncbi.nlm.nih.gov/pmc/articles/PMC252 8919/pdf/cjc220193.pdf

10. Barcelo A. Cardiovascular diseases in Latin America and the Caribbean. Lancet 2006; 368: 625-6. DOI: 10.1016/S0140-6736(06)692234.

11. Escoffery CT, Shirley SE. Causes of sudden natural death in Jamaica: a medicolegal (coroner's) autopsy study from the University Hospital of the West Indies. Forensic Sci Int 2002; 129: 116-21.

12. Madhavan SR, Reddy S, Panuganti P, Joshi R, Mallidi J, Raju K et al. Epidemiology of sudden cardiac death in rural south India - insights from the Andhra Pradesh rural health initiative [Internet]. Indian Pacing Electrophysiol J 2011 [cited 2014 Feb 3]; 11: 93-102. Available from http://www.ipej.org/1104/tandri.htm

13. Aligbe JU, Akhiwu WO, Nwosu SO. Prospective study of coroner's autopsies in Benin City, Nigeria. Med Sci Law 2002; 42: 318-24.

14. Morales A, Madrazo Y, Ramirez JI, Castaneda L, Machin W, Alvarez L et al. Acute myocardial infarction incidence, mortality and case fatality in Santa Clara, Cuba, 2007-2008 [Internet]. MEDICC Rev 2011 [cited 2014 Feb 3]; 13: 23-9. Available from: http://www.medicc.org/ mediccreview/index.php?issue $=18 \& \mathrm{id}=222 \& \mathrm{a}=\mathrm{va}$

15. Beharry A, Rios M, Sandy S, Chin J, Pooran S, Welch W et al. Audit of sudden deaths in the accident and emergency department of a tertiary hospital in Trinidad and Tobago [Internet]. West Indian Med J 2011 [cited 2014 Feb 3]; 60: 61-7. Available from: http://caribbean. scielo.org/scielo.php?script=sci_arttext\&pid=S0043-314420110 00100013

16. Zimmerman J, Fromm R, Meyer D, Boudreaux A, Wun C-C, Smalling $\mathrm{R}$ et al. Diagnostic marker cooperative study for the diagnosis of myocardial infarction [Internet]. Circulation 1999 [cited 2014 Feb 3]; 99: 1671-7. doi: 10.1161/01.CIR.99.13.1671. Available from: http://circ.ahajournals.org/content/99/13/1671.full.pdf html

17. Thygesen K, Searle, J. Update on the universal definition of acute myocardial infarction in the light of new data [Internet]. Conference Papers in Medicine 2013 [cited 2014 Apr 11]; 2013: 479352. Available from: http://dx.doi.org/10.1155/2013/479352

18. Smolina K, Wright FL, Rayner M, Goldacre MJ. Determinants of the decline in mortality from acute myocardial infarction in England between 2002 and 2010: linked national database study [Internet]. BMJ 2012 [cited 2014 Feb 3]; 344: d8059. doi: 10.1136/bmj.d8059. Available from: http://www.bmj.com/content/344/bmj.d8059

19. Ministry of Health, Trinidad and Tobago. Annual statistical report 2004-2005 [Internet]. Port-of-Spain: Ministry of Health; 2005. Available from: http://www.health.gov.tt/sitepages/default.aspx?id=122

20. Miller GJ, Maude GH, Beckles GLA. Incidence of hypertension and non-insulin dependent diabetes mellitus and associated risk factors in a rapidly developing Caribbean community: the St James survey, Trinidad. J Epidemiol Community Health 1996; 50: 497-504.

21. Thomas CN, Titus G, Williams D, Simeon D, Pitt-Miller P. Two-year mortality and its determinants following acute myocardial infarction in Trinidad and Tobago. West Indian Med J 2000; 49: 112-14.

Received 19 May 2014

Accepted 19 Jun 2014

Revised 03 Jun 2015

Published 01 Jul 2015

Online: http://www.mona.uwi.edu/wimjopen/article/1614

(C) Bahall and Seemungal 2015

This is an open access article made freely available under Creative Commons Attribution 4.0 International (CC BY 4.0). Users are free to share, copy and adapt this work as long as the copyright holder (author) is appropriately and correctly credited. See http://creativecommons.org/ licences/by/4.0/deed.en_us for more information. 